CLAWAR 2020: $23^{\text {rd }}$ International Conference on

Climbing and Walking Robots and the Support

Technologies for Mobile Machines,

Moscow, Russian Federation, 24-26 August 2020.

https://doi.org/10.13180/clawar.2020.24-26.08.10

\title{
SELECTION OF OPTIMAL PARAMETERS OF THE PNEUMATIC / HYDRAULIC ACTUATOR
}

\author{
KIRILL TRUKHANOV \\ Bauman Moscow State Technical University, Address: \\ Moscow, 105005, Russia, ul. Baumanskaya 2-ya, 5 \\ E-mail: trukhanov_ka@bmstu.ru, \\ https://bmstu.ru
}

\begin{abstract}
In modern fields of engineering and process control, pneumatic / hydraulic actuators are increasingly used. The effective use of pneumatic/ hydraulic actuators depends on their parameters, characteristics and properties. The methods of optimization are used to select the optimal actuator parameters, based on the minimum function search. The purpose of this article was the possibility of using the one decisive criterion (target function) that provides the optimal parameters of the pneumatic / hydraulic actuator with the translational motion of the output link, as well as its use at the synthesis of the servo pneumatic cylinder. To calculate the parameters of the servo pneumatic drive, close to optimal, it was used the $\mathbf{L P}_{\boldsymbol{\tau}}$-search with two criteria. It is shown and concluded that the proposed target function can be used at the synthesis of the servo pneumatic / hydraulic drives with the translational motion of the output link.
\end{abstract}

Nowadays, an increasing quantity of technological operations are being performed by robots and remotely controlled robotic systems. So, in many fields of technology, it is impossible to do without robotic systems at all. An example of such application of a robotic system is the developed device for repairing nuclear fuel storage pools at nuclear power plants (NPP), shown on Figure 1.

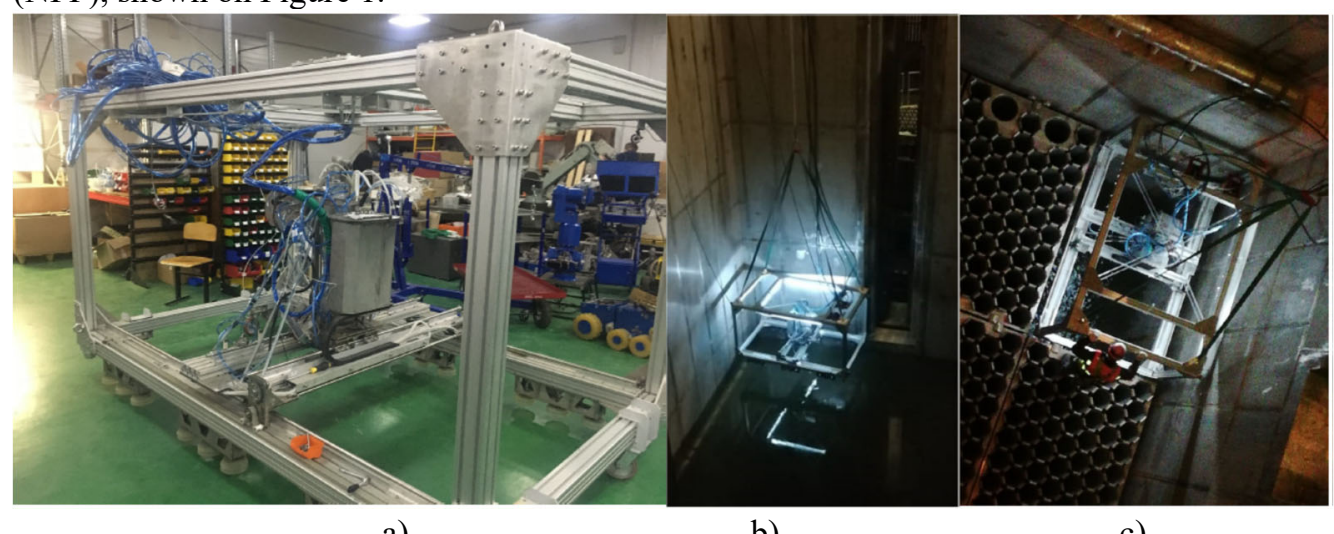

a)

b)

c)

Figure.1. A system for repairing pools at nuclear power plants. The system includes created servo pneumatic drives

a) - General view of the system for repairing pools at nuclear power plants before testing;

b) - system during tests at nuclear power plants;

c) - the system after testing at the nuclear power plant.

The remotely controlled robotic system (Fig.1) is a frame structure, the frame of which consists of rectangular aluminum profiles, on which the working tools of the manipulator are fixed: a welding head, an ultrasonic flaw detector and a sander. Also, on the frame of the device 
are located servo pneumatic drives that drive it. The servo pneumatic drives are either cylinders with a translational type of movement of the output link, or pneumatic motors - with a rotational movement of the output link. The actuators for moving the output links use the energy of compressed air supplied from the pneumatic system of the NPP. Thus, to determine the defect on the surface of the storage pool and then obviate it, it is necessary to have a system of tracking pneumatic devices that can move the process equipment along the found trajectory. Systems operating under unpredictable loads are required to find the places of defects in the NPP holding area with minimal positioning error and obviate them, since such defects lead to liquid leaks through the storage pool lining and are "the beginning of catastrophes".

The scientific novelty of the work is the developed method of selecting the optimal parameters of servo pneumatic drives at their design, which allows to create a drive design with optimal parameters without conducting expensive tests, and sometimes simply impossible tests, reducing the time and money spent on its development.

The selection of the parameters of the mechanisms and the basic scheme of the pneumatic / hydraulic actuator (synthesis) is a multicriteria task [1]. The operational efficiency of the pneumatic / hydraulic actuator depends on the technical parameters of the elements included in it, as well as from (as well as on) the accurate determination of the characteristics of both individual elements $[2,3]$ and the system as a whole [4]. As follows from the analysis of publications on this topic, to improve the synthesis of the quality and design time of the servo pneumatic / hydraulic actuator is needed to base on the method of researching the parameter space (RPS) [5 - 8]. This method allows us to pose and solve applied multicriteria optimization problems in various fields. However, the difficulty of constructing feasible sets leads to the difficulty of solving the stated optimization problems, which, in turn, forces us to carry out the correction of the initial optimization problem. An analysis of the technical literature showed that for probing the parameter space in the RPS method in most cases they use $\mathrm{LP}_{\tau}$-search that makes it possible to take into account the specifics of the engineering optimization problem [4-6, 8]. Thus, the solution of the adjusted optimization problem makes it possible to obtain the Pareto set, which is called the solution of the multicriteria optimization problem, assuming that after approximating the resulting set, the problem will be finally solved that meets the requirements for the operation of the pneumatic / hydraulic actuator [6].

For operation of the actuator of the pneumatic / hydraulic actuator, both discrete and proportional control are used. The proportional type of control has wider possibilities for regulating and controlling the working process, which include:

- in automatic, continuous and accurate tuning of the system parameters (force or moment; speed (acceleration) of movement (rotation);

- in positioning by linear or angular coordinate;

- in energy efficiency, i.e. energy input can be reduced by controlling pressure and / or flow in accordance with current actuator requirements;

- to simplify the system (one proportional valve can replace several discrete devices, for example, a throttle and a distributor).

The purpose of the work is to develop a method for justifying the selection of parameters close to the optimal (the best) pneumatic / hydraulic actuator with the translational movement of the output link.

At solving the technical task of finding the best parameters for a pneumatic / hydraulic actuator, it is necessary to determine a design option for which the optimization criteria would be of minimum value. 


\section{The selection of optimization criteria}

In practice, when designing technical systems, weight and size indicators and energy characteristics of pneumatic / hydraulic actuator elements are widely used, given by manufacturers in their catalogs. To compare design options, it is better to use quality indicators in a dimensionless form, defined as some similarity numbers. In the case of a pneumatic / hydraulic actuator with translational movement of the output link, this number is determined by the formula $[5,6]$ :

$$
\mu_{\mathrm{mp}}=\frac{m \cdot v_{p}^{2}}{P_{p} \cdot l}
$$

where the indices $\mathrm{m}$ and $\mathbf{p}$ in the expression for $\mu_{\mathrm{mp}}$ indicate its relationship with the mass and the translational motion of the output link of the product, respectively; $m$ is the mass of the device together with compressed air (with the working fluid in the case of a hydraulic actuator). The values of the parameters in formula (1) depend on the design and dimensions of the pneumatic / hydraulic cylinder, so there is no way to indicate the relationship of these parameters with each other.

The mass of the actuator $\boldsymbol{m}$ can be estimated by the expression $m=\rho_{m} \cdot V_{c}$. Here $\rho_{m}$ is the density of the material from which the cylinder body is made; $V_{c}$ - the volume of the body of the pneumatic / hydraulic cylinder together with the covers: $V_{c} \sim \frac{\pi}{4} \cdot\left[D^{3} \cdot(p /[\sigma])^{0,5}+\left(D \cdot \frac{p}{[\sigma]}\right)^{2} \cdot l\right]$, where $p$ is the nominal pressure of the working fluid in the pneumatic / hydraulic actuator; $[\sigma]$ is the allowable stress for the material of the cylinder body; $D$ is the piston diameter; $l$ - stroke of the output link of the pneumatic / hydraulic cylinder (rod of the pneumatic / hydraulic cylinder).

The speed of the translational movement of the output link of the pneumatic / hydraulic actuator $v_{p}$ is determined as $v_{p}=\frac{Q_{a}}{\pi \cdot D^{2} / 4}$. Here $Q_{a}$ is the flow rate of compressed air (working fluid) in the actuator.

The maximum force acting on the output link of the pneumatic / hydraulic actuator $P_{\Pi}$ is determined by the area of the piston of the cylinder and the nominal pressure of the working fluid in the pneumatic / hydraulic actuator, namely: $P_{p}=\frac{\pi}{4} \cdot D^{2} \cdot p$.

Taking into account the indicated relations, expression (1) takes the view:

$$
\mu_{\mathrm{m} \Pi}=\frac{f(D, p, l) \cdot\left(\frac{Q_{a}}{\pi \cdot D^{2} / 4}\right)^{2}}{\frac{\pi}{4} \cdot D^{2} \cdot p \cdot l}
$$

where $f(D, p, l)=m$.

The set of variable parameters in expression (2) is conveniently represented in the following view [5]

$$
\boldsymbol{X}=\left(x_{0}, x_{1}, x_{2}, x_{3}\right),
$$

where $x_{0} \equiv D, x_{1} \equiv p, x_{2} \equiv l, x_{3} \equiv Q_{a}$.

Then expression (2) can be accepted as the first optimization criterion $F_{1}(X)$ and present as

$$
F_{1}(X)=\frac{\left[x_{0}^{3} \cdot\left(x_{1}\right)^{0,5}+\left(x_{0} \cdot x_{1}\right)^{2} \cdot x_{2}\right] \cdot x_{3}{ }^{2}}{\frac{\pi^{2}}{16} \cdot x_{0}{ }^{6} \cdot x_{1} \cdot x_{2}} \rightarrow \text { min. }
$$

The smaller the overall dimensions of the actuator elements, that is, a function of the view (4), the better of the pneumatic / hydraulic actuator [6]. 
To assess the energy efficiency of a pneumatic / hydraulic actuator, it is necessary to determine the power value developed by it.

In dimensional form, the power value developed by the actuator has the view

$$
N_{a c}=p \cdot Q_{a},
$$

or otherwise,

$$
N_{a c}(X)=x_{1} \cdot x_{3} .
$$

A function of the form (6) is a monotonic dependence that increases with increasing parameters $x_{1}$ and $x_{3}$, which can be represented in another view

$$
F_{2}(X)=\frac{1}{N_{a c}(X)} \rightarrow \min ,
$$

and accept as an optimization criterion.

For further study of the results of optimization of actuator parameters, expressions (4) and (7) must be presented in a dimensionless form [5], namely:

$$
\begin{gathered}
\overline{F_{1}(X)}=\frac{\left[\left(\frac{x_{0}}{x_{0 \max }}\right)^{3} \cdot\left(\frac{x_{1}}{x_{1 \max }}\right)^{0,5}+\left[\left(\frac{x_{0}}{x_{0 \max }}\right) \cdot\left(\frac{x_{1}}{x_{1 \max }}\right)\right]^{2} \cdot\left(\frac{x_{2}}{x_{2 \max }}\right)\right] \cdot\left(\frac{x_{3}}{x_{3 \max }}\right)^{2}}{\frac{\pi^{2}}{16} \cdot\left(\frac{x_{0}}{x_{0 \max }}\right)^{6} \cdot\left(\frac{x_{1}}{x_{1 \max }}\right) \cdot\left(\frac{x_{2}}{x_{2 \max }}\right)} \rightarrow \text { min, } \\
\overline{F_{2}(X)}=\frac{1}{\overline{N_{a c}(X)}}=\left(\frac{x_{1 \max }}{x_{1}}\right) \cdot\left(\frac{x_{3 \max }}{x_{3}}\right) \rightarrow \text { min. }
\end{gathered}
$$

Since two optimization criteria have been chosen to solve the problem of finding the best parameters for a pneumatic / hydraulic actuator, there is a multi-criteria optimization task [6 - 8].

\section{The solution of the problem of multicriteria optimization}

When determining the best parameters for the synthesis of a servo pneumatic / hydraulic actuator, we used the $\mathrm{LP}_{\tau}$ search with two criteria (8) [6].

For practical use of $\mathrm{LP}_{\tau}$-search, it is advisable to choose the number of points equal to $N^{\prime}=2^{v}[6]$.

Estimation of the ratio of volumes $\gamma=V_{G} / V_{P} \approx N / N^{\prime}\left(V_{G}\right.$ is the volume of the bounded domain $\mathrm{G}$, containing $N^{\prime}$ points, $V_{P}$ is the volume of the bounded region $\mathrm{P}$ containing $\mathrm{N}$ points) showed that, for conducted $N=4096$ tests, of which $N^{\prime}=785$ satisfied functional limitations, an increase in the number of test points does not improve the calculation results, and a smaller number of test points does not determine the optimal solution.

It is well known that it is impossible to achieve the extreme value of all optimization criteria at the same time, in the case under consideration - the minimum value of $\overline{F_{1}(X)}$ and $\overline{F_{2}(X)}$. The solution to the problem can only be some compromise that belongs to the Pareto set of the problem, that is, such a set of solutions in which all optimization criteria cannot be improved simultaneously [8]. Thus, the solution to the two-criteria optimization problem is the Pareto set.

After constructing a piecewise linear approximation of the Pareto front, the final solution to this problem, the authors consider the point $X_{L P \tau}$ lying on the Pareto front, which is located at the minimum distance from the coordinate origin - at a radius $r$ (see Figure 2). The value $r$ corresponds to a point in the space of dimensional parameters of the system pneumatic follower [5]. 


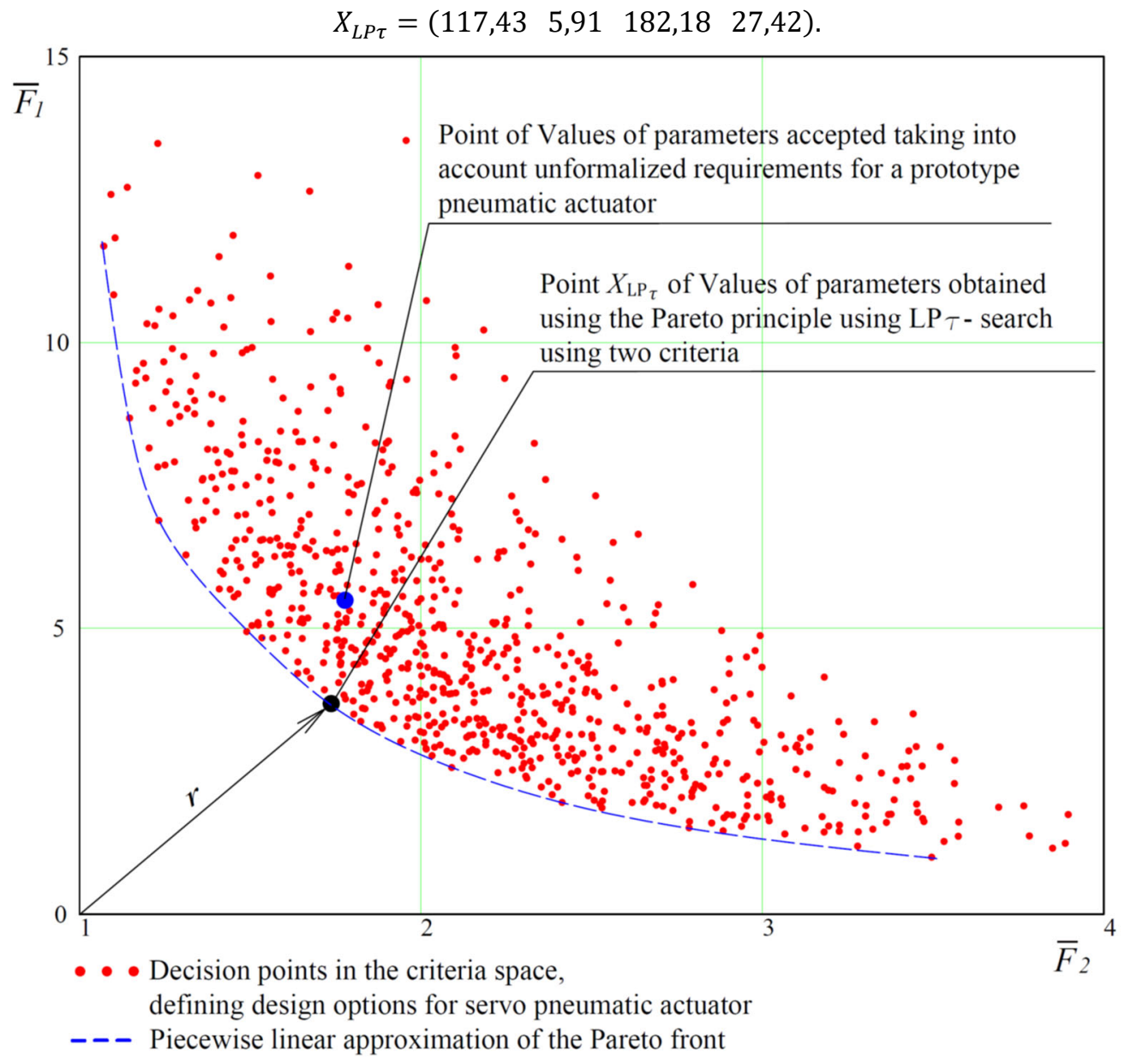

Figure.2. The results of determining the optimal parameters of the system with pneumatic actuator.

In addition to the selected two criteria of the form $\overline{F_{1}}$ и $\overline{F_{2}}$, when synthesizing a servo pneumatic drive, it is necessary to take into account unformalizable requirements arising from actual and commercially available actuator components. Therefore, the servo pneumatic drive was finally adopted with the parameters given in the table. 1.

Values of net power $N_{a c}$ pneumatic actuator for the parameters specified in table. 1 defined using formula (5) are given in table. 2.

Table 1. Parameters of the pneumatic follower

\begin{tabular}{lcccc}
\hline \multicolumn{1}{c}{ Parameters } & $D, \mathrm{~mm}$ & $p$, bar & $l, \mathrm{~mm}$ & $Q_{a}, \mathrm{lpm}$ \\
Method of determining & 117,43 & 5,91 & 182,18 & 27,42 \\
\hline $\begin{array}{l}\text { Values of parameters obtained } \\
\text { using the Pareto principle using }\end{array}$ & & & & \\
$\begin{array}{l}L P_{\tau^{-}} \text {search using two criteria } \\
\text { Values of parameters accepted } \\
\text { taking into account unformalized } \\
\begin{array}{l}\text { requirements for a prototype } \\
\text { pneumatic actuator }\end{array}\end{array}$ & 150,00 & 6,00 & 150,00 & 26,49 \\
\hline
\end{tabular}


Table 2. Net power of the pneumatic follower

\begin{tabular}{lc}
\hline \multicolumn{1}{c}{ The method of determining the value of power } & $\begin{array}{c}\text { The value of the power of the } \\
\text { pneumatic follower, } \mathrm{kW}\end{array}$ \\
\hline $\begin{array}{l}\text { The power value } N_{\mathrm{LP}_{\tau}} \text { obtained using the Pareto } \\
\text { principle using } L P_{\tau} \text { - search }\end{array}$ & 16,21 \\
\hline $\begin{array}{l}\text { Power value } N_{a c} \text { taken as a result of interactive design } \\
\text { of the pneumatic follower system }\end{array}$ & 15,89 \\
\hline
\end{tabular}

The maximum relative error of the obtained value of the useful power of the follower pneumatic actuator can be estimated by the value

$$
\Delta=\frac{\left|N_{\mathrm{LP}_{\tau}}-N_{a c}\right|}{N_{\mathrm{LP}_{\tau}}} \cdot 100 \%
$$

According to expression (9), the maximum relative error is $\Delta=1.92 \%$, which is an indicator that, under the assumptions made and the selected actuator parameters, the developed tracking pneumatic actuator system is close to the Pareto optimal, since the resulting error is less than 5\%. In addition, this indicator ensures the creation of an actuator from actuators devices standardly produced by famous industrial companies.

\section{Design features of the synthesized servo pneumatic cylinder}
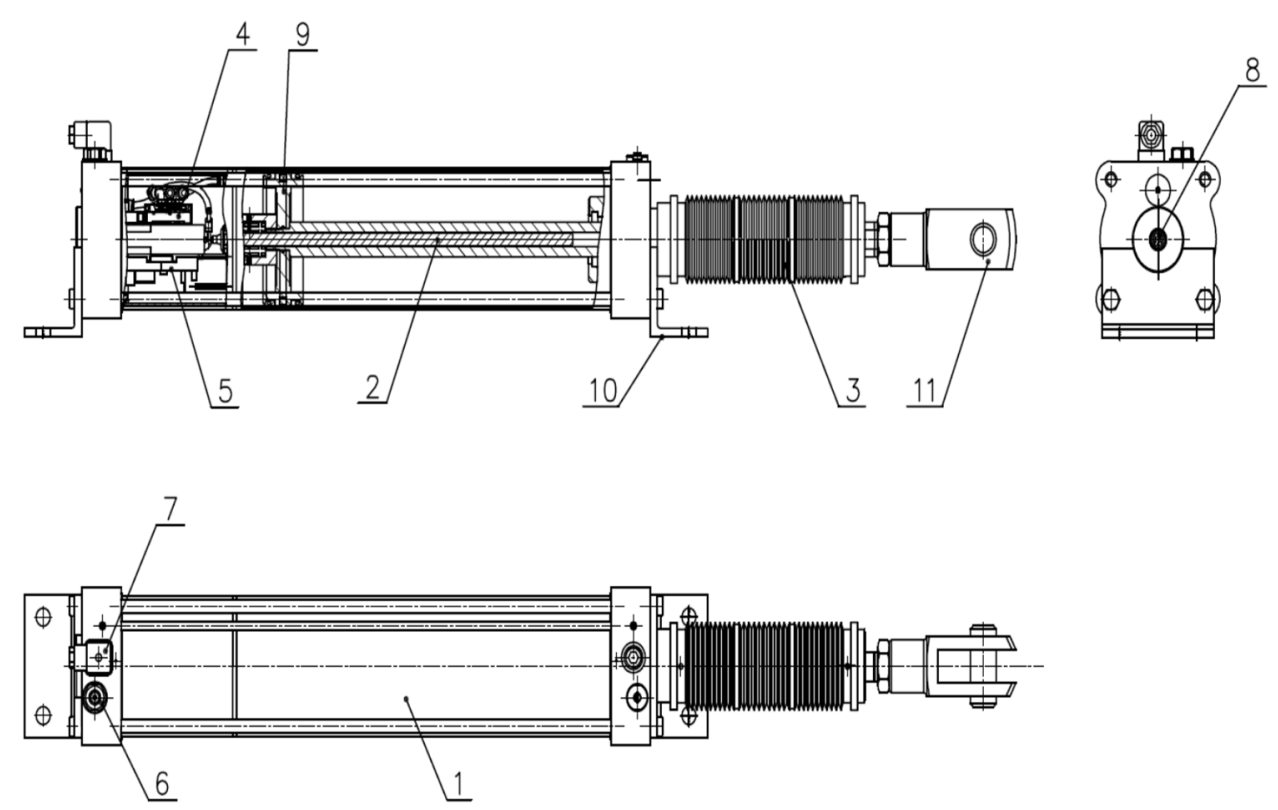

Figure.3. General view of a linear type pneumatic follower (servo cylinder) with an integrated control system ("hidden" circuit): 1 - pneumatic cylinder; 2 - feedback sensor; 3 - stock protection; 4 - the distributor; 5 - control unit; 6 - supply of compressed air; 7 - plug socket; 8 - service connector; 9 - a piston; 10 - mounting feet (bracket); 11 fork-shaped head. 
The follower pneumatic actuator (servo cylinder) (SPP) consists of a pneumatic cylinder 1 (Fig. 3) with the reciprocating movement of the output link (rod) (linear actuator) with a hollow rod [9-11]. Inside the rod there is a sensor 2 for the position of the rod, for example, for this version of the potentiometric type. Inside the SPP, there is a control unit 5 (controller) and a proportional valve 4 . This circuit is called "hidden". In addition to this design, where the control unit 5 and the distributor 4 are hidden inside the NGN, there is another variant of the NGN design - an "exploded" scheme in which the control unit 5 and the distributor 4 are located separately from the pneumatic cylinder 1. An example of this embodiment is shown in Figure 4.

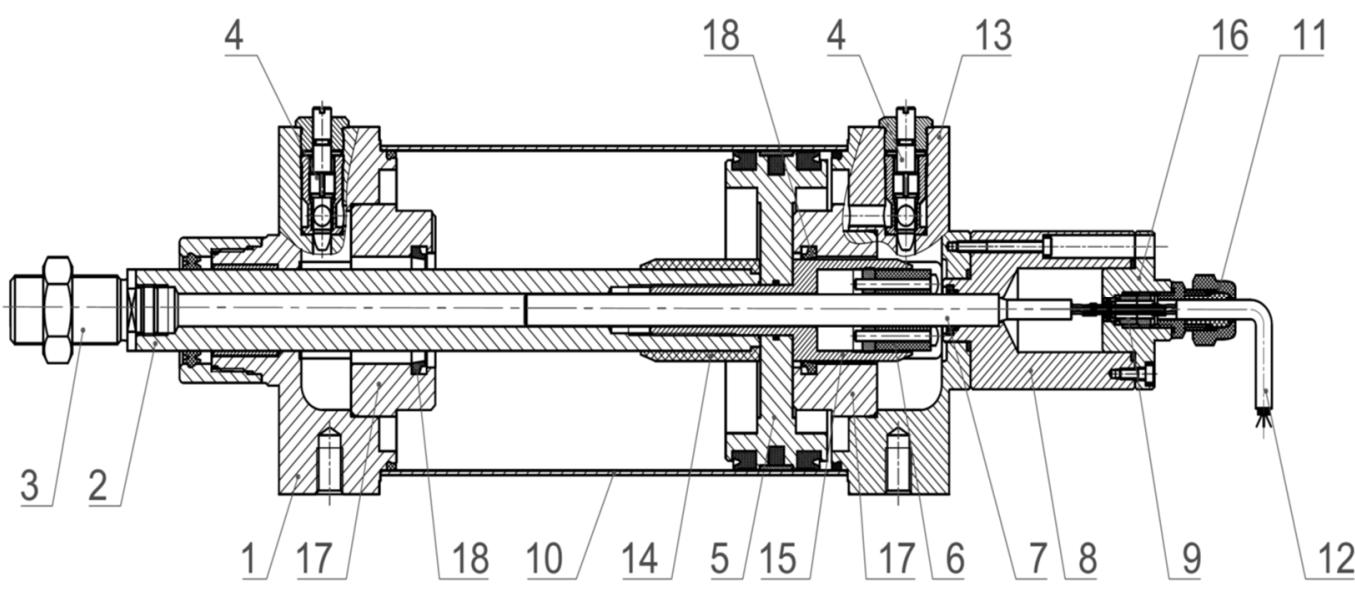

Figure.4. General view of a linear type pneumatic follower with an external control system ("spaced" circuit):

1 - front cover; 2 - hollow stock; 3 - tip (rod support); 4 - mechanical throttle end of stroke; 5 - the piston; 6 - magnetic coupling of the feedback sensor; 7 - feedback sensor (DOS); 8 - a protective casing of DOS; 9 - pressure seal; 10 sleeve (body) of the pneumatic cylinder; 11 - DOS wire protection; 12 - DOS connection wire; 13 - a back cover; 14 ,

15 - (guide) locking element of the throttle end of stroke; 16 - casing cover; 17 - cover sleeve; 18 - seal.

The advantages of an SPP scheme with a "hidden" control system are its compactness, the absence of additional lines connecting the distributor and the pneumatic cylinder, and ease of installation. The disadvantages of the scheme include the increased cost, the complexity of the repair structure. In contrast to the "hidden" scheme, the "diversity" scheme has a number of advantages: a simpler and more reliable design; ease of repair and lower cost. The disadvantages of the scheme include the presence of additional communication lines and large overall dimensions.

The static characteristic of the SPP is shown in Fig.5. For the stability of the SPP, its design uses a feedback sensor with an output current signal, i.e. the feedback signal varies from 4 to 20 $\mathrm{mA}$. This is necessary so that, firstly, electromagnetic pickups arising from the operation of technological equipment do not affect the operation of the control system of the SPP, and secondly, do not distort the control and feedback signals. Further, for convenience of working with this signal, this signal is converted into a voltage signal using a resistor, for example, with a nominal value of $250 \mathrm{Ohms}$. In view of this, the static characteristic shown in Fig.5 has its origin not at zero on the abscissa, but is shifted by $1.04 \mathrm{~V}$ (the deviation from $1 \mathrm{~V}$ is due to the error of the resistor), which corresponds to the value of $4 \mathrm{~mA}$ feedback sensor with the rod fully 
retracted. The real static characteristic - "2" is superimposed on the ideal - "1" with slight deviations (Fig.5).

\section{The static characteristic of the SPP}

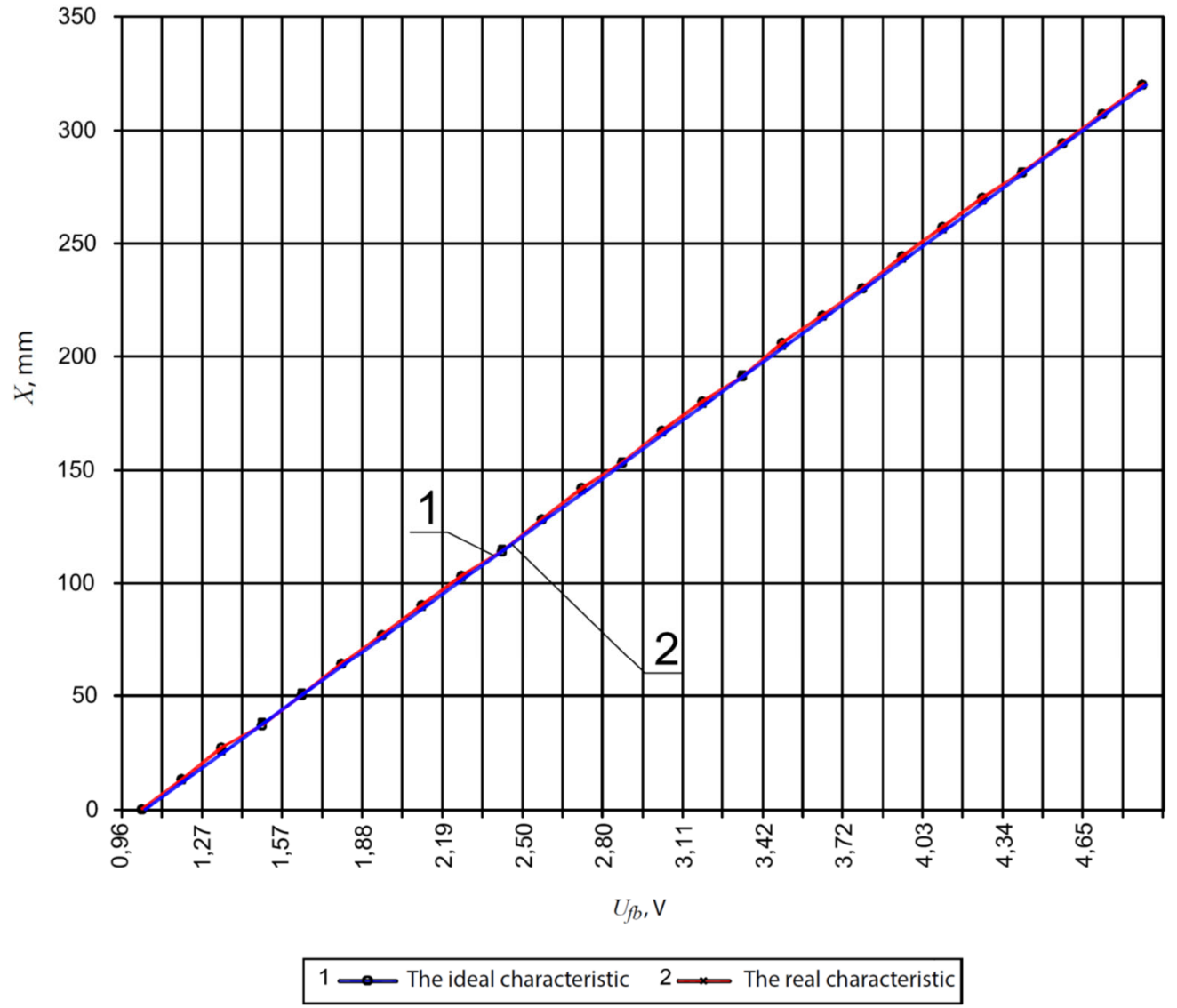

Figure.5. Static characteristic of the pneumatic follower (SPP): $x$ - position of the rod of the SPP; $U_{f b}$ - feedback

voltage.

\section{Conclusion}

The results of the work are as follows:

1. Optimization criteria (8) are formulated and presented that can be used in the synthesis of a servo pneumatic / hydraulic actuator, and which make it possible to make an informed choice of the best parameters of a pneumatic / hydraulic actuator with translational motion of the output link.

2. The problem of two-criteria optimization is solved taking into account the unformalized requirements for a servo pneumatic / hydraulic actuator.

3. The design features of the pneumatic cylinder with feedback on the position of the rod, which is part of the synthesized subsequent pneumatic actuator, are shown. Based on the solution of the problem, we can do the following conclusions.

The selected optimization criteria (8) can be applied to select the optimal design parameters of pneumatic / hydraulic actuators with translational movement of the output link.

The accepted values of the parameters $D, p, l$ and $Q_{a}$ differ from the found optimal ones for several reasons. The pressure value $\mathrm{p}$ was taken according to Table 1 , due to the fact that this 
pressure level is set as standard in all modern industrial pneumatic actuators. The diameter value $D$ is designed in such a way as to provide a safety factor for the developed effort equal to 1.6. The value for the stroke was taken to be less than optimal to make the pneumatic cylinder more compact.

The useful power of the pneumatic cylinder obtained during the design of the actuator, which differs from the optimal value by $1.92 \%$, ensures the creation of a pneumatic actuator from standard products manufactured by firms. Tests of a prototype of such a pneumatic actuator confirm the feasibility of its use.

The scientific novelty of the work lies in the formulation and solution of the problem of two-criteria optimization of the pneumatic / hydraulic actuator with the translational movement of the output link.

\section{List of references}

1. Trukhanov K.A. Synthesis of a hydraulic actuator with discretely controlled movement of the output link: author. dis. ... cand. tech. Sciences. - M., 2013. - 16 p.

2. Trukhanov K.A., Efremova K.D., Makarov I.V. Methodology for identifying the transfer function of pneumatic / hydraulic units. // News of MSTU "MAMI". 2016. No4 (30). S. $74-$ 81.

3. Zamaraev D.S., Popov D.N. Throttle-controlled electro-hydraulic follow-up actuator optimization concept. // Science and education. MSTU named after N.E. Bauman. 2013. No. 6. DOI: 10.7463 / 0613.0569281.

4. Korkodinov D.V., Popov D.N. Optimal design of a servo electro-hydraulic actuator with throttle control // Hydraulic machines, hydraulic actuators and hydropneumatic automation: proceedings of the All-Russian scientific and technical. conf. undergraduate and graduate students (Moscow, December 9, 2010). - M .: Publishing House MPEI, 2010.S. 124-128.

5. Trukhanov K.A., Popov D.N. Selection of optimal parameters for the hydraulic fan actuator for the vehicle engine cooling system. // Science and education. MSTU named after N.E. Bauman. Electron. journal 2013. No. 7. DDI: 10.7463 / 0713.0590873

6. Sobol IM, Statnikov RB The choice of optimal parameters in tasks with many criteria: textbook. manual for universities. - M.: Bustard, 2006 .-- 176 p.

7. Borovin G.K., Popov D.N. Multicriteria optimization of hydraulic systems: textbook. manual for universities. - M.: MSTU. N.E. Bauman. 2007 .-- 94 p.

8. Karpenko A.P. Modern search engine optimization algorithms. Algorithms inspired by nature: textbook. allowance. - M.: MSTU. N.E. Bauman, 2017. - 448 p.

9. Catalog: Linear actuators with integrated feedback sensor. Actuator control systems with integrated encoder. FestoAG \& Co.Kg, 2015 .-- 43 pgs.

10. James F. Blubaugh, Hans P. Dietz, Wayne W. Lark and other. Fluid cylinder with embedded positioning sensor. United States Patent: January 21, 2003. Patent Number No. 6,509,733 B2.

11. Vincent McCarroll, R. Howe, Blake Carter, Daniel Cook. Servo-pneumatic actuator. United States Patent: February 23, 2006. Patent Number No. US20060037467 A1. 